\title{
Stigmatizing attitudes toward people living with HIV/AIDS (PLWHA) among primary health care providers in Kinta District, Perak
}

\author{
Chan Yuen Ching, Maliza Mawardi, Adibah Hanim Ismail@Daud
}

Chan YC, Mawardi M, Adibah HI. Stigmatizing attitudes toward people living with HIV/AIDS (PLWHA) among primary health care providers in Kinta District, Perak. Malays Fam Physician. 2021;16(1);31-38. https://doi.org/10.51866/oa0001

\section{Keywords:}

Stigmatizing attitudes,

primary health care

providers, HIV, AIDS

\section{Authors:}

Maliza binti Mawardi

(Corresponding author)

MBBS (Malaya), MFamMed (Malaya)

Faculty of Medicine and Health

Science, Universiti Putra Malaysia

Malaysia

Email: maliza_mawardi@upm.edu.my

\section{Chan Yuen Ching}

MD (UPM) MMed FamMed (UPM)

Klinik Kesihatan Greentown

Ministry of Health, Malaysia.

\section{Adibah Hanim binti Ismail@}

Daud

MD (USM), MMed FamMed (USM)

Faculty of Medicine and Health

Science, Universiti Putra Malaysia

Malaysia

\section{Abstract}

Background: Stigmatizing attitudes expressed by health care providers prevent some members of at-risk populations from accessing human immunodeficiency virus (HIV) screening and care. This attitude contributes to the continuity of the infection dissemination within our community, which gives an impact on the healthcare service and the curtailment of the global HIV/acquired immunodeficiency syndrome (AIDS) pandemic.

Objective: This study was conducted to identify stigmatizing attitudes toward people living with HIV/AIDS (PLWHA) and their determinants among primary health care providers in Kinta District, Perak.

Methodology: A cross-sectional study was conducted in 36 primary care clinics in Kinta District, Perak. Using stratified random sampling, 365 primary health care providers were recruited into the study. A validated self-administered questionnaire was used to obtain sociodemographic data as well as information on the healthcare experiences of healthcare providers, their knowledge of HIV/AIDS, and attitudes toward PLWHA. Determinants were identified using multiple linear regression.

Results: More than half of the respondents (54.1\%) had never provided care to HIV/AIDS patients. A minority (29.9\%) had received training on HIV/AIDS. This study shows that doctors (Coef. $=-9.50,95 \% \mathrm{CI}:-18.93,-0.07, \mathrm{p}=0.048$ ), respondents with HIV-positive relatives, (Coef. $=-5.61,95 \% \mathrm{CI}:-10.57,-0.65, \mathrm{p}=0.027)$, those who had provided care to HIV/AIDS patients (Coef. $=-2.38,95 \%$ CI: $-4.31,-0.45, \mathrm{p}=0.016$ ), and those with a higher knowledge score on HIV/AIDS (Coef. $=-0.86,95 \% \mathrm{CI}:-1.59,-0.13, \mathrm{p}=0.021$ ) were less likely to show stigmatizing attitudes toward PLWHA.

Conclusion: The issue of stigmatizing attitudes toward PLWHA among primary health care providers needs to be addressed. This study finds that knowledge, profession, experiences with caring for PLWHA, gender, and having HIV-positive relatives are significant predictors of stigmatizing attitudes toward PLWHA among primary health care providers in Kinta District, Perak. Interventional programs to improve knowledge and awareness, as well as decrease stigma toward PLWHA, should be implemented among all health care providers, especially those who have no opportunity to provide direct care.

\section{Introduction}

Since the onset of the human immunodeficiency virus (HIV) epidemic in 1981, 75 million people have been diagnosed with HIV, and more than one-third of them die from HIV/acquired immunodeficiency syndrome (AIDS). ${ }^{1}$ The Joint United Nations Program on HIV and AIDS (UNAIDS) and the World Health Organization (WHO) have found that the primary reason most people are hesitant to receive HIV testing, reveal their HIV status, and obtain treatment is a fear of stigma and discrimination. ${ }^{2}$ Additionally, the People Living with HIV (PLHIV) Stigma Index interviewed PLHIV and aggregated data from 50 countries, finding that about $12.5 \%$ of them are turned down from health care services due to stigma and discrimination. ${ }^{3}$

Many studies conducted worldwide have identified factors that are associated with stigmatizing attitudes toward people living with HIV/AIDS (PLWHA) among health care workers, especially in countries where HIV is highly prevalent, such as in Nigeria and South Africa. 4,5 These factors vary among countries. The stigmatizing attitudes of health care providers may also vary depending on the place of practice, as hospital settings differ from primary health care settings. 
In Malaysia, anonymous HIV screening and HIV care have been provided in all government health clinics since the year 2000. The Prevention of Mother-To-ChildTransmission (PMTCT) Programme that screens all pregnant women for HIV began even earlier, in 1998, involving government health clinics. This screening programme was extended into community clinics (Klinik Komuniti) in 2015. All family medicine specialists (FMS) nationwide have been trained in HIV care, and many mobile CD4 point-ofcare tests are available at primary care clinics as of December 2015.6 Therefore, the attitudes of primary health care professionals toward PLWHA are important, as the stigmatizing attitudes they express may prevent members of at-risk populations from accessing HIV screening and treatment. ${ }^{7-10}$

It is important to understand the attitudes of primary health care providers toward PLWHA, as HIV patients increasingly seek treatment and follow-ups from FMS in government health clinics. The results of this study will contribute to a better understanding of the determinants of stigmatizing attitudes among primary health care providers, which could further be used in the development of interventions to improve attitudes based on the factors that contribute to stigmatization. Thus, health care services for PLWHA would indirectly improve with improvements in attitudes toward sufferers among health care providers.

\section{Method}

A cross-sectional study was conducted from July to August 2019 in primary care clinics in Kinta District, Perak. All health care providers who have had direct or indirect contact with PLWHA or patients who were suspected to have HIV were recruited for this study. This included doctors, nurses, medical assistants, pharmacists, health care assistants, medical laboratory technologists (MLT), and radiographers. The calculated sample size was 365, using the formula for comparing means between two independent groups, in accordance with a study by Waluyo et al. ${ }^{8}$ There are a total of 36 primary health care clinics under the Kinta District Health Office. These clinics include 14 Klinik Kesihatan, 5 Klinik Komuniti, 12 Klinik Desa, and 5 Klinik Kesihatan Ibu \& Anak (KKIA). Samples were selected using stratified random sampling from a list of all health care providers registered under this district health office, which consists of 164 doctors, 411 nurses, 81 medical assistants, 95 pharmacists, 87 health care assistants (PPK), 42 medical laboratory technologists (MLT), and 13 radiographers. The eligible individuals were listed according to their job strata, namely 1 . doctor, 2 . nurse, 3. medical assistant, 4. pharmacist, 5. health care assistant (PPK), 6. medical laboratory technologist, and 7. radiographer. The requisite proportion of each stratum in the district was calculated. Samples were then randomly selected using a random number generator to meet the calculated proportion.

\section{Data collection}

Health care providers were approached individually by the researcher. They were given an explanation and written information regarding the study. Informed and written consent was obtained from those who were willing to participate. The pretested questionnaire was provided to the participants and collected by the end of the day by the researcher to ensure completeness. Participants were kept anonymous, as no personal information was required in the questionnaire. All consent forms containing personal information were kept separately from the questionnaire.

\section{Study instrument}

The questionnaire consisted of four sections: 1) sociodemographic profile, 2) health care experiences, 3) HIV/AIDS knowledge, and 4) attitudes toward PLWHA. The sections on HIV/AIDS knowledge and attitudes toward PLWHA were adopted from a validated questionnaire by Vorasane et al. ${ }^{10}$ Permission from the authors in both sections were obtained. ${ }^{11,12}$ The HIV/AIDS knowledge section consists of 10 items that had previously been used in many HIV studies in China. ${ }^{11}$ Attitudes toward PLWHA were measured using 17 items as well as a fivefactor multidimensional HIV-related stigma scale, which was developed and validated using both exploratory and confirmatory factor analysis. ${ }^{12}$ The fit of the final confirmatory factor model with five subscales displayed excellence in both development and validation samples. The result of the confirmatory analysis between the five subscales and each item in the subscales was significant $(p<0.01){ }^{12}$ The Cronbach's alpha coefficient was reported to be $0.81,{ }^{10}$ a higher score indicating more severe 
stigmatizing attitudes. The questionnaires were translated from English to Malay by two bilingual Malaysians who are well versed in both languages. Back translation from Malay to English was performed by a different pair of bilingual Malaysians who were also well versed in both languages. The discrepancies of the translation were then discussed among the translators and researchers in order to come to an agreement on the final translation as well as ensure semantic and content validity. A pilot study was conducted among 40 health care providers in Hospital Pengajar Universiti Putra Malaysia (HPUPM) to assess the face validity of the Malay questionnaire. One question each in Sections 1, 2, and 4 was revised by changing the wording without changing the meaning based on the feedback obtained during the pilot study. The questionnaire distributed to the respondents was bilingual.

\section{Data analysis}

Statistical Package for Social Sciences (SPSS) version 20.0 was used to analyze the data. Before conducting a descriptive analysis, normality testing was performed for all continuous data. The results were reported as medians and interquartile ranges (IQR), as the data was not normally distributed. Multiple linear regression (MLR) was applied to factors with $p<0.25$ in bivariate analysis. The results of the MLR were presented as coefficients and $95 \%$ confidence intervals. The significance level was set at $p<0.05$.

Ethical clearance was obtained from the Medical Research and Ethics Committee (MREC) of the Ministry of Health, Malaysia.

\section{Results}

Out of 365 selected primary health care providers in Kinta District, 355 of them agreed to participate in this study and returned completed questionnaires, creating a response rate of $97.3 \%$.

The median age of the respondents was 36.0 years, with an IQR of 8 . The majority of the respondents were female $(81.1 \%)$, Malay $(74.1 \%)$, married $(86.8 \%)$, and had completed at least tertiary education (91.8\%). A small percentage $(3.1 \%)$ of the respondents had relatives with HIV infection. Almost half of the respondents were nurses $(46.8 \%)$, which aligns with the proportion of health care providers in PKD Kinta. More than half of the respondents (54.1\%) had never provided care to HIV/ AIDS patients. A minority of respondents $(29.9 \%)$ had received training on HIV/AIDS.

Table 1: Sociodemographic profiles and health care experiences of respondents

\begin{tabular}{|l|c|c|}
\hline \multirow{2}{*}{ Variable } & \multicolumn{2}{|c|}{ N $=355$} \\
\cline { 2 - 3 } & Frequency (n) & \\
\hline Age (years) (median 36.0, IQR 8) & \multicolumn{2}{|c|}{} \\
\hline Gender & \multicolumn{2}{|c|}{} \\
Male & 67 & 18.9 \\
Female & 288 & 81.1 \\
\hline Ethnicity & & \\
Malay & 263 & 74.1 \\
Chinese & 31 & 8.7 \\
Indian & 58 & 0.9 \\
Others & 3 & 8.2 \\
\hline Educational level & & 44.8 \\
Secondary education & 29 & 15.8 \\
Certificate/diploma & 159 & 31.2 \\
Post-basic & 56 & \\
Degree & 111 & 86.8 \\
\hline Marital status & & 2.0 \\
Single & 38 & 0.5 \\
Married & 308 & \\
Divorced & 7 & \\
Widowed & 2 & \\
\hline
\end{tabular}




\begin{tabular}{|l|c|c|} 
Have relatives with HIV & & 3.1 \\
Yes & 11 & 96.9 \\
No & 344 & \\
\hline Profession & 63 & 17.7 \\
Doctor & 166 & 46.8 \\
Nurse & 33 & 9.3 \\
Medical assistant & 39 & 11.0 \\
Pharmacist & 32 & 9.0 \\
Health care assistant (PPK) & 17 & 4.8 \\
Medical laboratory technologist & 5 & 1.4 \\
Radiographer & & \\
Years of working experience (median 11.0, IQR 8) & & 45.9 \\
\hline Provided care to HIV/AIDS patients & 163 & 54.1 \\
Yes & 192 & \\
No & & 29.9 \\
\hline Training on HIV/AIDS & 106 & 70.1 \\
\hline Yes & 249 & \\
No & & \\
\hline
\end{tabular}

In this study, the minimum knowledge score was 2 and the maximum score was 10 . The median score was 9 with an IQR of 2 . The median score of stigmatizing attitudes was 45 with an IQR of 11, and scores ranged between 17 and 71.

\section{Factors associated with stigmatizing attitudes of primary health care providers toward PLWHA}

Bivariate analysis demonstrates that respondents with degrees (Coef. $=-8.43,95 \%$ confidence interval $[\mathrm{CI}]:-11.87,-4.99, p=<0.001$ ), health care providers who have HIV-positive relatives (Coef. $=-5.70,95 \%$ CI: $-11.05,-0.35$, $p=0.037)$, those who have provided care to HIV/AIDS patients (Coef. $=-4.90,95 \% \mathrm{CI}$ : $-6.70,-3.10, p=<0.001)$, and those with higher levels of HIV/AIDS knowledge (Coef. $=-1.51,95 \%$ CI: $-2.21,-0.81, p=<0.001)$ were less likely to display stigmatizing attitudes toward PLWHA. Furthermore, doctors (Coef. $=-15.58,95 \%$ CI: $-23.08,-8.09, p=<0.001)$, nurses (Coef. $=-7.45,95 \%$ CI: $-14.77,-0.13$, $\mathrm{p}=0.046$ ), medical assistants (Coef. $=-9.56$,
95\% CI: $-17.30,-1.82, p=0.016)$, and pharmacists (Coef. $=-10.38,95 \%$ CI: -18.04 , $-2.72, p=0.008)$ were less likely to demonstrate stigmatizing attitudes toward PLWHA. Female respondents were significantly associated with stigmatizing attitudes toward PLWHA (Coef. = 3.29, 95\% CI: 0.93, 5.65, $p=0.006$ ).

Multiple linear regression revealed that respondents who work as doctors (Coef. = $-9.50,95 \%$ CI: $-18.93,-0.07, p=0.048$ ), those who have HIV-positive relatives (Coef. = -5.61, 95\% CI: $-10.57,-0.65, p=0.027)$, those who have provided care to PLWHA (Coef. = $-2.38,95 \%$ CI: $-4.31,-0.45, p=0.016)$, and those with higher levels of knowledge on HIV/ AIDS (Coef. $=-0.86,95 \%$ CI: $-1.59,-0.13, p=$ $0.021)$ were less likely to manifest stigmatizing attitudes toward PLWHA. Female respondents were significantly associated with stigmatizing attitudes toward PLWHA (Coef. $=3.02$, 95\% CI: $0.34,5.71, p=0.028$ ). In this study, there was no multicollinearity found between the independent variables in which the tolerance values were more than 0.1 and variance inflation factor (VIF) values were less than 5 . 
Table 2: Factors associated with stigmatizing attitudes toward PLWHA among respondents (bivariate and multiple linear regression analysis)

\begin{tabular}{|c|c|c|c|c|c|c|}
\hline \multirow{2}{*}{ Characteristics } & \multicolumn{3}{|c|}{ Bivariate } & \multicolumn{3}{|c|}{ Multivariate } \\
\hline & Coef. & $95 \% \mathrm{CI}$ & $p$-value & Coef. & $95 \% \mathrm{CI}$ & $p$-value \\
\hline Age & 0.09 & $-0.05,0.23$ & 0.211 & $<0.001$ & $-0.34,0.34$ & 0.999 \\
\hline $\begin{array}{l}\text { Gender (Male as RC) } \\
\text { Female }\end{array}$ & 3.29 & $0.93,5.65$ & 0.006 & 3.02 & $0.34,5.71$ & 0.028 \\
\hline $\begin{array}{l}\text { Ethnicity } \\
\text { (Others as RC) } \\
\text { Malay } \\
\text { Chinese } \\
\text { Indian }\end{array}$ & $\begin{array}{l}1.52 \\
-2.16 \\
-3.64\end{array}$ & $\begin{array}{l}-8.44,11.48 \\
-12.53,8.21 \\
-13.79,6.52\end{array}$ & $\begin{array}{l}0.765 \\
0.682 \\
0.482\end{array}$ & & & \\
\hline $\begin{array}{l}\text { Educational level } \\
\text { (Secondary education as RC) } \\
\text { Certificate/diploma } \\
\text { Post-basic } \\
\text { Degree }\end{array}$ & $\begin{array}{l}-1.77 \\
-3.22 \\
-8.43\end{array}$ & $\begin{array}{l}-5.10,1.57 \\
-7.00,0.55 \\
-11.87,-4.99\end{array}$ & $\begin{array}{c}0.298 \\
0.094 \\
<\mathbf{0 . 0 0 1}\end{array}$ & $\begin{array}{c}0.02 \\
0.20 \\
-2.95\end{array}$ & $\begin{array}{l}-7.71,7.75 \\
-7.90,8.30 \\
-12.52,6.62\end{array}$ & $\begin{array}{l}0.996 \\
0.961 \\
0.544\end{array}$ \\
\hline $\begin{array}{l}\text { Marital status } \\
\text { (Widowed as RC) } \\
\text { Single } \\
\text { Married } \\
\text { Divorced }\end{array}$ & $\begin{array}{l}3.08 \\
5.04 \\
7.21\end{array}$ & $\begin{array}{l}-9.66,15.81 \\
-7.42,17.49 \\
-6.86,21.29\end{array}$ & $\begin{array}{l}0.635 \\
0.427 \\
0.314\end{array}$ & & & \\
\hline $\begin{array}{l}\text { Have HIV-positive relatives } \\
\text { (No as } \mathrm{RC} \text { ) } \\
\text { Yes }\end{array}$ & -5.70 & $-11.05,-0.35$ & 0.037 & -5.61 & $-10.57,-0.65$ & 0.027 \\
\hline $\begin{array}{l}\text { Profession } \\
\text { (Radiographer as RC) } \\
\text { Doctor } \\
\text { Nurse } \\
\text { Medical assistant } \\
\text { Pharmacist } \\
\text { Health care assistant } \\
\text { Medical lab technologist }\end{array}$ & $\begin{array}{l}-15.58 \\
-7.45 \\
-9.56 \\
-10.38 \\
-4.79 \\
-3.08\end{array}$ & $\begin{array}{l}-23.08,-8.09 \\
-14.77,-0.13 \\
-17.30,-1.82 \\
-18.04,-2.72 \\
-12.55,2.96 \\
-11.29,5.12\end{array}$ & $\begin{array}{l}<\mathbf{0 . 0 0 1} \\
\mathbf{0 . 0 4 6} \\
\mathbf{0 . 0 1 6} \\
\mathbf{0 . 0 0 8} \\
0.225 \\
0.461\end{array}$ & $\begin{array}{l}-9.50 \\
-6.34 \\
-5.74 \\
-5.66 \\
-4.45 \\
-3.39\end{array}$ & $\begin{array}{l}-18.93,-0.07 \\
-13.67,0.99 \\
-13.73,2.25 \\
-15.17,3.85 \\
-14.60,5.70 \\
-11.57,4.80\end{array}$ & $\begin{array}{l}\mathbf{0 . 0 4 8} \\
0.090 \\
0.158 \\
0.243 \\
0.389 \\
0.416\end{array}$ \\
\hline Years of working experience & 0.14 & $-0.002,0.28$ & 0.053 & 0.27 & $-0.32,0.37$ & 0.880 \\
\hline $\begin{array}{l}\text { Provided care to HIV/AIDS } \\
\text { patients (No as RC) } \\
\text { Yes }\end{array}$ & -4.90 & $-6.70,-3.10$ & $<0.001$ & -2.38 & $-4.31,-0.45$ & 0.016 \\
\hline $\begin{array}{l}\text { Training on HIV/AIDS } \\
\text { (No as RC) } \\
\text { Yes }\end{array}$ & -1.51 & $-3.54,0.52$ & 0.145 & -1.09 & $-3.03,0.85$ & 0.269 \\
\hline HIV/AIDS knowledge & -1.51 & $-2.21,-0.81$ & $<0.001$ & -0.86 & $-1.59,-0.13$ & 0.021 \\
\hline
\end{tabular}

$\mathrm{RC}=$ Reference category

\section{Discussion}

This study found that over half (54.1\%) of the respondents had never provided care to HIV/ AIDS patients. This finding aligns with those of similar studies conducted in Indonesia and Bangladesh, ${ }^{9,15}$ which could be due to the fact that all three studies included supporting staff in the study population, and only certain health care providers are directly involved in the management of HIV patients in a primary care setting. This includes FMS, medical officers, and nurses who are trained to handle HIV patients. This study demonstrates that only $29.9 \%$ of respondents had undergone training in terms of courses or workshops on HIV/AIDS. This contradicts the findings of another local study performed among nurses in a tertiary center, where $73.3 \%$ of respondents had been trained in HIV/AIDS. ${ }^{13}$ This reflects the inadequacy of training among primary health care providers in the Kinta 
district. The median knowledge score among the respondents was 9. This aligns with the findings of a study conducted in Lao PDR, where the same questionnaire on HIV/AIDS knowledge was used. ${ }^{10}$ However, the median knowledge score among the subgroup of health care assistants (PPK), medical lab technicians, and radiographers was lower (8), most likely because these professionals do not have direct contact with PLWHA and are not given the opportunity to attend courses related to HIV/ AIDS.

In this study, we aimed to determine the level of stigmatizing attitudes and their predictors among primary health care professionals toward PLWHA. Overall, the median score of stigmatizing attitudes in this study was high (45) and exceeded the score found in the study in Lao PDR, where the median score was $39 .{ }^{10}$ This signifies that primary health care providers in the Kinta district displayed greater stigmatizing attitudes toward PLWHA than those in Lao PDR. Factors associated with stigmatizing attitudes include gender, having HIV-positive relatives, profession, experiences with care of HIV patients, and HIV/AIDS knowledge.

Female respondents were more likely to display stigmatizing attitudes toward PLWHA, a finding that is consistent with those of a study conducted by Andrewin and Chien, who postulated that females tend to be more homophobic compared to their male counterparts. ${ }^{14}$ Women experience greater social consequences for behavior that is deemed to be sexually or morally deviant by the community. Most studies found that gender was not significantly associated with stigmatizing attitudes. ${ }^{8-10,15,16}$ However, all of these studies were conducted in a hospital setting.

Health care providers who have relatives with HIV displayed lesser stigmatizing attitudes toward PLWHA. This could be explained by their familiarity with the disease and low levels of perceived danger, as similarly shown in a study performed among relatives of patients with psychiatric illness. ${ }^{17}$ However, a study in Bangladesh showed that having relatives with HIV does not significantly influence stigmatizing attitudes among health care professionals. $^{9}$

In this study, it was found that doctors, compared to other health care providers, display lesser stigmatizing attitudes toward
PLWHA. Similar results have been found in other studies. ${ }^{9} 15,16,18$ Harapan et al. state that doctors have more HIV/AIDS knowledge compared to nurses and thus hold lesser stigmatizing attitudes toward PLWHA. ${ }^{15}$ In this study, doctors were also found to possess higher knowledge scores compared to other health care providers, thus displaying lesser stigmatizing attitudes toward PLWHA.

Health care providers who have provided care to PLWHA demonstrated lower stigma scores compared to those who have never cared for this group of patients. Other studies have also demonstrated that health care providers who have had direct physical contact with PLWHA display lesser stigmatizing attitudes. ${ }^{10,15,18}$ This could be due to contact with PLWHA mitigating stigmatizing attitudes and dispelling misconceptions regarding HIV infection and transmission. $^{18}$

Previous studies have shown that knowledge is one of the primary predictors of stigmatizing attitudes toward PLWHA. ${ }^{10,14-16,18}$ Our study illustrates that primary health care professionals with higher levels of HIV/AIDS knowledge displayed less stigma toward PLWHA. Greater education on HIV modes of transmission and universal precautions significantly contributes to the reduction of stigmatized attitudes toward PLWHA and instills more positive attitudes toward PLWHA in learners. ${ }^{15,19}$

This study has a few limitations. Firstly, this cross-sectional study was unable to demonstrate the causal relationship between the dependent variable and independent variables. Secondly, the results of the study cannot be generalized or applied to other settings, as they are confined to primary health care providers in one district in Perak. Moreover, the unequal gender ratio in this study affected the significance of the variable in the inferential analysis, which in turn resulted in female respondents being significantly associated with stigmatizing attitudes, a result that cannot be generalized. This ratio reflects a known scenario in health care settings in which females predominate most categories of health care personnel, especially among allied health staff professions, namely nurses and pharmacists. ${ }^{20}$ In terms of the study instrument, the knowledge and attitudes questionnaire used has yet to be validated locally. Therefore, it is recommended that future studies be carried out to develop a valid questionnaire determining the stigmatizing 
attitudes of health care providers toward PLWHA that could be used locally. Lastly, calculations regarding stigmatizing attitudes toward PLWHA reflect what the respondents reported. As some of the questions were deemed sensitive in nature, there is a possibility that answers provided by respondents were more socially acceptable than truthful. Hence, stigmatizing attitudes could be underreported in this study.

This study is the first to determine levels of stigmatizing attitudes toward PLWHA among primary health care providers in Malaysia. This study illustrates that lower levels of HIV/AIDS knowledge are associated with greater levels of stigmatizing attitudes toward PLWHA. Future studies may be conducted during the pre- and post-continuous medical education (CME) section on HIV/AIDS to demonstrate improvements in knowledge and attitudes. The CME session should target all levels of staff, not only doctors, nurses, and pharmacists but professionals that have indirect contact with HIV patients as well, including health care assistants, medical laboratory technicians, and radiographers. Furthermore, this study indicates that only $29.9 \%$ of health care providers have had training on HIV/AIDS. Another study suggests that attending a short course can improve knowledge, and thus, providing short courses on HIV/AIDS may able to improve the knowledge of health care providers and lower levels of stigma toward PLWHA. ${ }^{21}$

\section{Conclusion}

The issue of stigmatizing attitudes toward PLWHA among primary health care providers must be addressed. This study finds that knowledge, profession, experiences with care of PLWHA, gender, and having relatives with HIV are significant predictors of stigmatizing attitudes toward PLWHA among primary health care providers in Kinta District, Perak. Interventional programs to improve knowledge and awareness regarding stigma toward PLWHA should be implemented among all health care providers, especially those who have no opportunity to provide direct care.

\section{Acknowledgments}

We would like to acknowledge the Director General of Health Malaysia for his permission to publish this article. The authors wish to thank Pegawai Kesihatan Daerah Kinta and health care providers in Kinta District for providing support during data collection. We also express gratitude to Madam Premaa Supramaniam for her assistance in the statistical analysis of the study.

\section{Conflicts of interest}

The authors declared that they have no conflicts of interest.

\section{How does this paper make a difference to general practice?}

- The lack of training of primary health care providers in HIV/AIDS indicates that more training should be provided to improve their knowledge and thus improve attitudes toward PLWHA as well as those who come in for screening.

- The negative relationship between HIV/AIDS knowledge and stigmatizing attitudes toward PLWHA indicates that continuous medical education (CME) should be provided to all levels of primary health care providers, including health care assistants, medical lab technologists, and radiographers, as they have indirect contact with HIV patients as well.

\section{References}

1. WHO $\mid$ HIV/AIDS [Internet]. WHO. [cited 2019 Sept 25]. Available from: https://www. who.int/gho/hiv/en/
2. United Nations Development Programme (UNDP) US. 2014unaidsguidancenote_ stigma_en.pdf [Internet]. [cited 2018 Jul 19]. (Reduction of HIV-related stigma and discrimination). Available from: http:// www.unaids.org/sites/default/files/media_ asset/2014unaidsguidancenote_stigma_en.pdf
3. Developing tools and methods to measure HIV-related stigma and discrimination in health care settings in Thailand. International Health Policy Program Ministry of Public Health, Thailand, 2014. Available from: http:// pdf.usaid.gov/pdf_docs/PA00KHKM.pdf 
4. Umeh CN, Essien EJ, Ezedinachi EN, et al. Knowledge, Beliefs and Attitudes about HIV/AIDS related issues, and the Sources of Knowledge among Health Care Professionals in Southern Nigeria. J R Soc Promot Health. 2008 Sep;128(5):233-9

5. Okpala PU, Uwak R, Nwaneri AC, et al. Nurses' knowledge and attitude to the care of HIV/AIDS patients in South East, Nigeria. Int J Community Med Public Health. 2017 Jan 25;4(2):547-53.

6. HIV/STI Section of Ministry of Health Malaysia editorial team. Global AIDS Response Progress Report Malaysia 2016. 2016;40.

7. Wagner AC, Hart TA, McShane KE, et al. Health Care Provider Attitudes and Beliefs About People Living with HIV: Initial Validation of the Health Care Provider HIV/ AIDS Stigma Scale (HPASS). AIDS Behav. 2014 Dec 1;18(12):2397-408

8. Waluyo A, Culbert GJ, Levy J, et al. Understanding HIV-related stigma among Indonesian nurses. J Assoc Nurses AIDS Care JANAC. 2015;26(1):69-80.

9. Hossain MB, Kippax S. Stigmatized Attitudes Toward People Living With HIV in Bangladesh: Health Care Workers' Perspectives. Asia Pac J Public Health. 2011 Mar 1;23(2):171-82.
10. Vorasane S, Jimba M, Kikuchi K, et al. An investigation of stigmatizing attitudes toward people living with HIV/AIDS by doctors and nurses in Vientiane, Lao PDR. BMC Health Serv Res [Internet]. 2017 Feb 10 [cited 2018 Jan 28];17. Available from: https://www.ncbi. nlm.nih.gov/pmc/articles/PMC5301416/

11. Li L, Wu ZY, Wu S, et al. HIV-related stigma in health care settings: A survey of service providers in China. AIDS Patient Care STDs. 2007;21(10):753-62

12. Stein JA, Li L. Measuring HIV-related Stigma Among Chinese Service Providers. AIDS Behav. 2008 Sep;12(5):789-95.

13. Gulifeiya A, Rahmah MA. Nurses awareness and attitude toward HIV/AIDS and universal precautions: a cross-sectional study in UKMMC. J Kesihat Masy. 2008;14(2):36-45.

14. Andrewin A, Chien L-Y. Stigmatization of patients with HIV/AIDS among doctors and nurses in Belize. AIDS Patient Care STDs. 2008 Nov;22(11):897-906

15. Harapan H, Khalilullah SA, Anwar S, et al. Discriminatory attitudes toward people living with HIV among health care workers in Aceh, Indonesia: A vista from a very low HIV caseload region. Clin Epidemiol Glob Health. 2015 Apr 1;3(1):29-36.
16. Famoroti TO, Fernandes L, Chima SC. Stigmatization of people living with HIV/AIDS by healthcare workers at a tertiary hospital in KwaZulu-Natal, South Africa: a cross-sectional descriptive study. BMC Med Ethics. 2013 Dec 19;14(Suppl 1):S6.

17. Sousa S de, Marques A, Rosário C, et al. Stigmatizing attitudes in relatives of people with schizophrenia: a study using the Attribution Questionnaire AQ-27. Trends Psychiatry Psychother. 2012;34(4):186-97.

18. Librarian I. Evaluation of Knowledge, Attitudes, and Practices of Health Care Providers toward HIV-Positive Patients in Tanzania [Internet]. [cited 2018 May 21]. Available from: https://www.hrhresourcecenter. org/node/1747.html

19. Zarei N, Joulaei H, Darabi E, et al. Stigmatized Attitude of Healthcare Providers: A Barrier for Delivering Health Services to HIV Positive Patients. IJCBNM. 2015;3(4):292-300.

20. Planning Division, Ministry of Health, Malaysia. Human Resources for Health, Country Profiles 2015, Malaysia

21. Josep M.A, Gemma.F, Josep J, et al. Effectiveness of a short-course in improving knowledge and skills on evidence-based practice. BMC Family Practice volume 12, Article number: 64 (2011) 


\section{Knowledge, attitude, and practice regarding atrial fibrillation among primary care physicians: the potential role of postgraduate training}

Phaik Choo Ooi, Gogilavendan Ramayah, Syahril Rizwan B. Omar, Vilasini Rajadorai, Thatsheila Nadarajah, Chung Hui Ting, Cheong Lieng Teng

Ooi PC, Ramayah G, Syahril RO, et al. Knowledge, attitude, and practice regarding atrial fibrillation among primary care physicians: the potential role of postgraduate training. Malays Fam Physician. 2021;16(1);39-49. https://doi.org/10.51866/oa0002

\section{Keywords:}

Knowledge, attitude, and practice; primary care physicians; atrial fibrillation; anticoagulation

\section{Authors:}

\section{Ooi Phaik Choo}

(Corresponding author) MBBS (UM), MAFP/FRACGP

Community Based Department Universiti Kuala Lumpur, Royal College of Medicine Perak Ipoh, Perak, Malaysia email: Ivkopc@yahoo.com

Gogilavendan Ramayah MBBS, MAFP/FRACGP Buntong Health Clinic Ipoh, Perak, Malaysia

Syahril Rizwan B. Omar MBBS

Simee Health Clinic

Ipoh, Perak, Malaysia

Vilasini Rajadorai

MBBS

Ayer Tawar Health Clinic

Ayer Tawar, Perak, Malaysia

Thatsheila Nadarajah

$M D$

Menglembu Health Clinic

Ipoh, Perak, Malaysia

\section{Abstract}

Introduction: Atrial fibrillation (AF) is known to lead to stroke and thromboembolism, causing a five-fold increase in the risk of stroke and almost doubling the mortality rate. Optimal anticoagulant therapy is effective in reducing AF-related death. However, prescription of anticoagulants in $\mathrm{AF}$ in East Asian countries has been low, ranging from $0.5 \%$ to $28 \%$. This study aimed to determine whether vocational training in family medicine improves primary care physicians' knowledge, attitude, and practice in the management of AF.

Method: This investigation was a cross-sectional study carried out during centralized workshops for two groups of trainees using a validated questionnaire: (i) junior trainees were newly enrolled postgraduate trainees in the Graduate Certificate in Family Medicine (GCFM) program, and (ii) senior trainees were postgraduate trainees in Advance Training in Family Medicine (ATFM) programs of the Academy of Family Physicians of Malaysia (AFPM).

Results: A total of 223 trainees (127 junior and 96 senior) participated in this study. Only 55.2\% of the trainees passed the knowledge test; senior trainees were more likely to pass the knowledge test compared to junior trainees $(69.8 \%$ vs. $44.1 \%$, p < 0.001$)$. Female trainees were significantly more likely to pass the knowledge test than male trainees. While the attitude of senior and junior trainees was similar, more of the latter group worked in public clinic that provide better support where there is better support for outpatient anticoagulation treatment (e.g., same-day INR test, direct access echocardiogram, and warfarin in in-house pharmacy).

Conclusion: Vocational training in family medicine appears to improve primary care physicians' knowledge regarding the management of AF. Better knowledge will help vocationally trained primary care physicians to provide anticoagulation treatment for AF within primary care clinics. More optimal AF management within primary care can take place if the identified barriers are addressed and a shared care plan can be implemented.

\section{Introduction}

Atrial fibrillation (AF) is the commonest cardiac arrhythmia of clinical significance. ${ }^{1-3}$ It causes systemic thromboembolism and is associated with a five-fold increased risk of stroke. ${ }^{4,5}$ Moreover, $15 \%$ of all strokes are related to $\mathrm{AF}^{2}$ Ischemic strokes associated with $\mathrm{AF}$ are typically more severe than those from other causes, resulting in a doubled risk of death and higher morbidity. ${ }^{5}$

The prevalence of AF varies from one population to another. In the general population, the average prevalence of $\mathrm{AF}$ is between $1 \%$ to $2 \% .{ }^{1}$ However, the prevalence varies, progressively increasing with age, reaching $10 \%$ to $17 \%$ among those $>80$ years old. ${ }^{1}$ Furthermore, this figure is likely underestimated, as $10 \%$ to $40 \%$ of $\mathrm{AF}$ patients can be asymptomatic and undiagnosed. ${ }^{1}$ Asymptomatic AF patients have a similar or higher risk of stroke and death compared to symptomatic patients. ${ }^{1}$

In Malaysia, the AF prevalence among hospitalized patients was $2.8 \% .^{6,7}$ In UK primary care, the age-sex standardized prevalence of $\mathrm{AF}$ increased $50 \%$ from $2.14 \%$ in 2000 to $3.29 \%$ in $2016 .{ }^{4}$ This trend is in keeping with estimates that the prevalence of AF will at least double in the coming 50 years due to an aging population. $^{2}$

Oral anticoagulation (OAC) with vitamin $\mathrm{K}$ 


\author{
Ting Chung Hui \\ MD, MAFP/FRACGP \\ Gunung Rapat Health Clinic \\ Ipoh, Perak, Malaysia
}

\section{Teng Cheong Lieng}

MFamMed (UM), FRACGP, FAMM.

Professor

Department of Family Medicine

International Medical University

Seremban Campus, Seremban

Negeri Sembilan, Malaysia antagonist (VKAs) or novel oral anticoagulants (NOACs) can markedly reduce the risk of stroke by approximately two-thirds. ${ }^{4}$ In comparison, aspirin use is considerably inferior in preventing stroke while presenting a similar risk of major bleeding. ${ }^{4}$ Unfortunately, despite the availability of clinical practice guidelines and evidence of the effectiveness of anticoagulation therapy in stroke prevention, many studies have confirmed gross underuse of anticoagulant therapy in patients with $\mathrm{AF}^{6-8}$

In Asia, the reported prevalence of stroke related to $\mathrm{AF}$ in community- and hospital-based studies has ranged from $1.9 \%$ to $6.0 \%$ and $0.36 \%$ to $28.3 \%$, respectively. ${ }^{8}$ However, anticoagulation prescription in AF in East Asian countries has been low, ranging between $0.5 \%$ and $28 \%{ }^{7}$ Globally, most studies have shown treatment rates of $\leq 50 \%$, even in hospital-based studies. ${ }^{6,10}$

Freestone et al. $^{6}$ found that patients with $\mathrm{AF}$ were not adequately investigated. The researchers' findings also asserted that the use of anticoagulation therapy was suboptimal among acute medical admissions to a busy hospital in Malaysia. Only $20 \%$ of the patients were treated with warfarin; among them, none were aged $>65$ years. ${ }^{6}$

Studies have identified three general categories of barriers to the prescription of anticoagulants, especially warfarin: i) physician-related factors, ii) patient-related factors, and iii) healthcare system-related factors. ${ }^{9,10}$

The physician-related barriers include significant knowledge gaps $^{9-11}$ and physicians' perceptions regarding the risks and benefits of anticoagulation and practice characteristics, which determine their attitude and practice in the management of AF.9-13

In Malaysia, the Academy of Family Physicians of Malaysia (AFPM) offers a postgraduate program consisting of four years of vocational training in family medicine. The first two years comprise the Graduate Certificate in Family Medicine (GCFM), and the last two years involve Advanced Training in Family Medicine (ATFM). Both courses include a cardiovascular module.

AF imposes a substantial burden on the future cost of health care. ${ }^{5}$ Primary care physicians can potentially play a larger role in the management of patients with AF. Thus, both determining their knowledge regarding AF and assessing their readiness to manage $\mathrm{AF}$ are vital.

The primary objective of this study is to compare the knowledge, attitudes, and practices between senior ATFM trainees and junior (newly registered) GCFM trainees in the management of AF. In particular, we wish to identify specific knowledge gaps and suggest a focus for future educational intervention.

\section{Methods}

\section{Study participants and setting}

This cross-sectional study was conducted from May to August 2017. The study participants were postgraduate trainees of the Academy of Family Physicians Malaysia (AFPM). We regarded the first group as junior trainees (newly enrolled GCFM trainees who had not undergone the training) and the second group as senior trainees (having completed two cardiology modules, which included diagnosis and management of atrial fibrillation).

\section{Questionnaire development and standard- setting}

Self-administered validated structured questionnaires were used to collect data. The questionnaires comprised four sections.

Section A: Demographic data, including age, gender, ethnicity, years of working as a doctor, years of working in primary care, current workplace, location of practice (urban, suburban, or rural), and current level of training. Section B: Knowledge test comprised of ten single-best answer multiple-choice questions, based on Malaysian and international clinical practice guidelines and consisted of five clinical vignettes (Appendix 1).

Section C: Assessment of participants' attitude in the management of AF. This section presented five questions about the opinion of the doctors regarding the diagnosis and management of atrial fibrillation, to be answered on a Likert scale of 1 (strongly disagree) to 6 (strongly agree) Section D: Assessment of participants' practice in the management of AF. This part consisted of five questions regarding the current practice of the doctors in the diagnosis and management of AF, to be answered by choosing from three options: Yes, Not sure, or No.

Sections C \& D were developed based on a 
literature review. No formal validation was done. The content of the knowledge test was developed using the Delphi technique, and standard-setting was conducted using the Nedelsky method. The standard was set at the level of a practicing family physician. This process involved an expert panel of nine members, consisting of three academic family physicians, a family medicine specialist at a government health clinic, a general practitioner, three academic internists, and one general physician. The passing mark based on the standard setting was $55 \%$.

A pilot study was conducted with ten medical officers at various public primary care clinics in Perak. The above group completed the questionnaire within 20 minutes. Because they did not encounter any difficulty in answering, no amendment was necessary.

Data collection was conducted during the lunch or tea breaks of the centralized workshops (unrelated to the management of AF) organized by AFPM in July 2017 (GCFM intake 18 and ATFM intake 6) and August 2017 (ATFM intake 5) with AFPM approval. Informed consent was obtained from the participants before data collection, and a pen was distributed to each participant as a token of appreciation.

Contact email addresses were requested from study participants who wanted to receive feedback about their performance on the knowledge test. The email addresses were not recorded in the data file and were deleted from the sender's email account immediately after the knowledge test results were sent to the respective participants.

\section{Statistical analysis}

The collected data were analyzed using IBM SPSS Statistical Software version 25. The outcome variables comprised scores on the knowledge test and responses to the attitude and practice questions. The independent variables included trainee category (junior or senior), workplace, practice location, gender, age, and duration of working in primary care.

The knowledge score was calculated as a proportion of correct answers for the tenitem MCQs, in percentages. A passing score was defined as a score of $55 \%$ and above. The overall summative scores and the percentage of participants who answered correctly for individual questions were also tabulated.

Responses to the attitude questions were based on a 6-point Likert-type scale ranging from 1 (strongly disagree) to 6 (strongly agree). Median scores were computed for the responses to each question because the data were not normally distributed.

The responses to the practice questions were recoded as follows: a "Yes" response remained as it was, while "Not sure" and "No" were merged into another category. The percentages of the responses for each question were computed.

The proportion who passed the knowledge test was compared using a chi-square test. Logistic regression was performed to determine the variables independently associated with pass/fail in the knowledge test. The knowledge scores of the two groups of trainees were compared using the Mann-Whitney $U$ test. Furthermore, the Mann-Whitney $U$ test and chi-square test were used to compare differences in the responses of the two groups of trainees for the attitude and practice questions, respectively. Statistical significance was set at $\mathrm{p}<0.05$.

\section{Results}

Out of 265 eligible trainees, 223 (127 junior trainees and 96 senior trainees) participated, giving a response rate of $84.2 \%$. The sociodemographic characteristics of the study participants show a predominance of females, urban practice locations, and working in public primary care clinics (see Table 1). The mean ages of junior and senior trainees were 31.8 and 35.9 years, respectively. 
Table 1: Demographic data of study participants

\begin{tabular}{|l|c|c|c|}
\hline & Junior trainees (n, \%) & Senior trainees (n, \%) & P-value \\
\hline Gender & $36(28.3)$ & $27(28.1)$ & 0.971 \\
Fale & $91(71.7)$ & $69(71.9)$ & \\
\hline Ethnicity & & & \\
Malay & $49(38.6)$ & $29(30.2)$ & 0.265 \\
Chinese & $38(29.9)$ & $40(41.7)$ & \\
Indian & $33(26.0)$ & $24(25.0)$ & \\
Others & $7(5.5)$ & $3(3.1)$ & 0.022 \\
\hline Workplace & $103(81.1)$ & $65(67.7)$ & \\
Public clinic & $21(16.5)$ & $25(26.0)$ & \\
Private clinic & $3(2.4)$ & $6(6.2)$ & \\
Other clinics* & & & \\
\hline Practice location & $73(57.5 \%)$ & $60(62.5 \%)$ & \\
Urban & $37(29.1 \%)$ & $31(32.3 \%)$ & \\
Suburban & $17(13.4 \%)$ & $5(5.2 \%)$ & \\
Rural & & & \\
\hline Years working in & & $48(0 \%)$ & \\
primary care & $11(8.7 \%)$ & $4(45.8 \%)$ & \\
$<1$ & $8(6.3 \%)$ & 96001 \\
$1-5$ & $3(2.4 \%)$ & & \\
6-10 & 127 & & \\
$>10$ & & & \\
\hline Total & & & \\
\hline & & & \\
\hline
\end{tabular}

* Other clinics include primary care clinics at universities, military health facilities, etc.

\section{Knowledge of atrial fibrillation}

Overall, a total of $123(55.2 \%)$ out of 223 trainees passed the knowledge test; senior trainees were significantly more likely to pass than junior trainees $(69.8 \%$ vs. $44.1 \%, \mathrm{p}<0.001)$. In the univariate analysis, three other variables were found to be statistically associated with passing the knowledge test: female gender (59.4\% vs. $44.4 \%)$ and the number of years working as a doctor and in primary care (see unadjusted OR in Table 2).

No significant difference in the knowledge score was found between doctor's age and practice locations.

In our logistic regression model, which included four variables, only senior trainees and the female gender remained statistically significantly associated with passing the knowledge test. See Table $\mathbf{2}$.

For the summative scores of the knowledge test, the overall mean was $56.10 \%$ (Table 3). The mean score for the junior and senior trainees was $51.0 \%$ and $62.8 \%$, respectively $(\mathrm{p}=<0.001)$. Thus, the seniors performed significantly better than the juniors.

Table 2: Factors associated with passing atrial fibrillation knowledge test

\begin{tabular}{|l|c|c|}
\hline Variables & Unadjusted OR (95\%CI) & Adjusted OR (95\%CI) \\
\hline Category of trainees (senior vs. junior) & $2.93(1.68,5.12)$ & $2.59(1.35,5.00)$ \\
\hline Gender (female vs. male) & $1.83(1.01,3.29)$ & $2.01(1.07,3.75)$ \\
\hline Years working as a doctor & $1.08(1.01,1.16)$ & $1.04(0.94,1.15)$ \\
\hline Years working in primary care & $1.12(1.02,1.23)$ & $1.00(0.96,1.15)$ \\
\hline
\end{tabular}


For individual questions, the percentage of participants who answered correctly was tabulated (Table 3).

Table 3: Knowledge test: percentage of correct answers

\begin{tabular}{|l|c|c|c|c|}
\hline Knowledge questions & $\begin{array}{c}\text { Overall } \\
\mathbf{( \% )}\end{array}$ & $\begin{array}{c}\text { Junior } \\
\text { trainees } \\
\mathbf{( \% )}\end{array}$ & $\begin{array}{c}\text { Senior } \\
\text { trainees } \\
\mathbf{( \% )}\end{array}$ & P-value \\
\hline 1. Management of AF in valvular heart disease & $56.5 \%$ & $58.3 \%$ & $54.2 \%$ & 0.541 \\
\hline 2. Management of lone AF & $43.5 \%$ & $33.9 \%$ & $56.3 \%$ & 0.001 \\
\hline 3. CHA2DS2-VASc scoring & $58.7 \%$ & $49.6 \%$ & $70.8 \%$ & 0.001 \\
\hline 4. Patient with TIA on aspirin & $56.5 \%$ & $59.1 \%$ & $53.1 \%$ & 0.376 \\
\hline 5. Factors affecting bleeding risks & $74.0 \%$ & $69.3 \%$ & $80.2 \%$ & 0.066 \\
\hline 6. Target of INR & $76.2 \%$ & $72.4 \%$ & $81.3 \%$ & 0.126 \\
\hline 7. Dietary advice for warfarin & $48.9 \%$ & $36.2 \%$ & $65.6 \%$ & $<0.001$ \\
\hline 8. Investigations for AF & $74.9 \%$ & $68.9 \%$ & $83.3 \%$ & 0.011 \\
\hline 9. Management strategy in AF & $21.1 \%$ & $22.1 \%$ & $19.8 \%$ & 0.683 \\
\hline 10. Drug choice for rate control & $53.4 \%$ & $43.3 \%$ & $66.7 \%$ & 0.001 \\
\hline Total & $56.1 \%$ & $51.0 \%$ & $62.8 \%$ & $<0.001$ \\
\hline
\end{tabular}

The senior trainees showed better knowledge than junior trainees for almost all the questions, reaching statistical significance for five questions (Q2, Q3, Q7, Q8, Q10). We noted that the performance of both junior and senior trainees was relatively poor for Q9.

\section{Attitude}

Regarding the attitude toward the management of AF, no significant difference was notable between the two groups in the responses to 4 out of 5 questions asked (Table 4). However, junior trainees were significantly more likely to agree that "routine screening of AF in the elderly is useful."

Table 4: Median score of responses to attitude questions

\begin{tabular}{|l|c|c|c|}
\hline Attitude questions & Junior trainees & Senior trainees & P-value \\
\hline 1. Routine screening of AF in the elderly is useful. & 5 & 4 & $<0.001$ \\
\hline $\begin{array}{l}\text { 2. AF can be managed competently in primary } \\
\text { care setting. }\end{array}$ & 5 & 4 & 0.817 \\
\hline 3. Warfarin has too many diet-drug interactions. & 4 & 4 & 0.743 \\
\hline $\begin{array}{l}\text { 4. Warfarin causes more harm than good in } \\
\text { elderly aged }>75 \text { years. }\end{array}$ & 4 & 4 & 0.345 \\
\hline $\begin{array}{l}\text { 5. Patients with AF in primary care are not keen } \\
\text { on warfarin therapy. }\end{array}$ & 3 & 4 & 0.297 \\
\hline
\end{tabular}

\section{Practice}

Our study showed that the availability of resources for AF management was relatively low in primary care clinics (see Table 5). Interestingly, we found that junior trainees answered "yes" to four of the five practice questions compared to the senior trainees.
Significantly more junior trainees reported availability of direct access echocardiography, same-day INR within the clinic, and warfarin available in the clinic pharmacy. Regarding counseling of warfarin patients about fooddrug interactions, junior trainees were significantly more likely to perform this task than senior trainees (Table 5). 
Table 5: Responses to practice questions

\begin{tabular}{|l|c|c|c|c|}
\hline Questions & $\begin{array}{c}\text { Overall } \\
\mathbf{( \% )}\end{array}$ & $\begin{array}{c}\text { Junior } \\
\text { trainees } \\
\mathbf{( \% )}\end{array}$ & $\begin{array}{c}\text { Senior } \\
\text { trainees } \\
\mathbf{( \% )}\end{array}$ & P-value \\
\hline $\begin{array}{l}\text { 1. I can request echocardiography without going } \\
\text { through the specialist clinic. }\end{array}$ & 48.4 & 56.7 & 37.5 & 0.005 \\
\hline $\begin{array}{l}\text { 2. My clinic can perform INR within the same } \\
\text { day. }\end{array}$ & 29.6 & 38.6 & 17.7 & 0.001 \\
\hline 3. Warfarin is available in my clinic pharmacy. & 48.0 & 55.1 & 38.5 & 0.014 \\
\hline $\begin{array}{l}\text { 4. I would adjust warfarin dosage for AF patients } \\
\text { if needed. }\end{array}$ & 68.6 & 72.4 & 63.5 & 0.156 \\
\hline $\begin{array}{l}\text { 5. I routinely advise warfarin patients about diet } \\
\text { and drug interactions. }\end{array}$ & 71.7 & 81.1 & 59.4 & $<0.001$ \\
\hline
\end{tabular}

\section{Discussion}

The results of this study showed an overall pass rate of $55.2 \%$ in the knowledge test, indicating that a significant knowledge gap remains among both groups of postgraduate family medicine trainees regarding the management of AF. This finding concurs with previous studies that also identified a significant knowledge gap among physicians in relation to $\mathrm{AF}$ and stroke. ${ }^{11}$ In fact, educational gaps were found across the continuum of care for AF patients. ${ }^{14}$ This result could be due to physicians being unaware of the current literature ${ }^{9}$ or overestimating the efficacy of antiplatelet agents or overestimating the risk of hemorrhage from anticoagulation. ${ }^{11}$

Besides the above educational gaps, while factual content on AF might receive adequate coverage in the vocational training syllabus for the trainees, the knowledge was likely not reinforced in the trainees' daily clinical practices. Many of them do not treat AF patients regularly due to a lack of resources and the nature of their practices, as highlighted by our study. Interventions that include interdisciplinary case discussions and seminars focusing on $\mathrm{AF}$ management during the vocational training may be helpful in this aspect.

By comparing the two groups, our study identified specific knowledge gaps among the senior trainees from the questions where they performed worse or not significantly better than the junior trainees (Q1, Q4, Q5, Q6, \& Q9). These areas included the management of AF in valvular heart disease, a TIA patient on aspirin, factors affecting bleeding risk, the target INR, and the management strategy in AF.

In particular, Q9 (on the management strategy in $\mathrm{AF}$ ) performance reflected gaps in the knowledge of senior trainees, representing a crucial area that requires further education and/ or management in consultation with specialists.

Multiple previous studies have recommended educating physicians as a possible solution to improve the management of $\mathrm{AF}^{11,13}$ It is reassuring that senior trainees showed a higher pass rate on the AF knowledge test compared to the junior trainees. Future study is needed to demonstrate whether better knowledge of senior trainees will translate into better management of AF patients.

Both groups of trainees in our study had a similar attitude regarding the management of AF, except in their answers to Q1. Compared to the seniors, the junior trainees were significantly more likely to acknowledge the usefulness of screening for AF in the elderly (see Table 4) ( $<$ 0.001). This outcome was in contrast with the recommendation by the Malaysian Ministry of Health for opportunistic case finding for AF with radial pulse palpation $(\geq 20 \mathrm{sec})$ during BP screening ${ }^{15}$ and a similar recommendation by the 2016 ESC guidelines for the management of $\mathrm{AF}$ to screen patients aged $\geq 65$ years. $^{16}$

Our study showed that the senior trainees had less access to resources for the management of $\mathrm{AF}$ in their practices compared to the junior trainees. This scenario could be the barrier that contributed to the poorer attitude that the senior trainees exhibited. Due to the inconvenience of referral to a secondary care center for management, they might have underestimated a patient's ability to comply with the management ${ }^{11}$ in the event that the screening found AF in an elderly patient. Of 
course, this outcome could alternatively be due to unawareness of the current literature by the participants. However, it was also likely that despite their knowledge, they underestimated the benefits of anticoagulation for stroke prevention and overestimated the harm of major bleeding with anticoagulation. ${ }^{13}$ These factors could lead to the perception and attitude that screening for AF is not very useful. Thus, further studies are required to determine the fundamental factors that caused the physicians to not comply with the clinical management guidelines in local settings in order to address the barriers in physicians' attitudes effectively.

Unexpectedly, the junior trainees revealed significantly better practice in the management of AF compared to the senior trainees. This outcome could be due to the fact that significantly more junior trainees worked at public clinics compared to the senior trainees (see Table 1), offering greater availability of same-day INR testing, warfarin in the clinic pharmacy, and direct access to an echocardiogram in the public clinics. Practice factors (e.g., availability of the INR test, anticoagulants that can be prescribed by family physicians) present major barriers to upgrading AF management in Malaysian primary care clinics. This issue demands the attention of the relevant policymakers.

As in our study, numerous other studies have also recognized physicians' negative beliefs and attitudes as major obstacles against anticoagulations ${ }^{10-12}$ and acknowledged healthcare system barriers ${ }^{9,10}$ Patient-related barriers is another vital aspect that requires analysis. ${ }^{9,10}$

Further studies to assess the knowledge, attitude, and practice of the same subjects before and after vocational training should also be carried out to provide a better reflection of the effect of vocational training.

Many of the stable AF patients who presented at the emergency care of the Mayo Clinic in Rochester, MN (USA), were seen for long-term follow-up by the specialists at the secondary care level rather than at the primary care level. ${ }^{17}$ The situation is similar in Malaysia, leading to congestion at the specialists' clinics.

The 2016 European Society of Cardiology (ESC) guidelines on the management of AF have recommended an integrated approach as a solution to overcome the increasing burden of AF-related health care (Recommendation Class IIa, Level of evidence B). ${ }^{16}$ Along the same line, Gallagher et al. ${ }^{18}$ found that integrated care improved patient outcomes in the management of AF by reducing allcause mortality and hospitalization related to cardiovascular conditions.

The benefit of integrated care was reaffirmed by van den Dries et al. in a randomized controlled trial on integrated care for elderly AF patients in primary care that demonstrated a significant $45 \%$ decrease in all-cause mortality compared to conventional care. ${ }^{19}$ In their study, besides focusing on team-based integrated care involving general practitioners, nurses, and specialists, the authors also emphasized the importance of training providers. ${ }^{19}$

Therefore, to optimize the care of patients with AF, Malaysia should consider exploring the option of implementing integrated care that involves primary care providers, particularly trained family physicians.

The evaluation and management of AF are fairly complicated and frequently not straightforward. ${ }^{14}$ Thus, wider implementation of AF management in primary care settings requires careful consideration regarding the training of the primary care doctor (especially for many who are not trained in family medicine) as well as provision of supportive services (echocardiogram, INR tests, medications, and consultation with a specialist) to both the public and private primary care sectors.

It is also vital for educational intervention programs, including vocational training in family medicine, to incorporate activities that will help translate knowledge on AF into practice in the management of AF. ${ }^{14}$

\section{Conclusion}

Vocational training in family medicine may improve primary care physicians' knowledge of the management of AF. However, the currently available training has not improved their attitude and practices due to multiple obstacles. Our study identified barriers involving both physicians and the healthcare system to the provision of warfarin management for atrial fibrillation in 
Malaysian primary care settings. The study also identified specific areas of knowledge gaps and the need to translate knowledge into practice, which can comprise a focus for future educational intervention.

\section{Acknowledgements}

The authors would like to express their sincere gratitude and appreciation to the following:

1. Academy of Family Physicians of Malaysia and postgraduate trainees

2. The Medical Ethics Committee of the International Medical University for giving the ethics approval and a small grant for this study (Project ID: IMU 384/2017)

3. Members of the expert panel involved in the validation of the knowledge test:
Prof. Dr. Esha Das Gupta (Internist \& Rheumatologist, IMU Seremban), Dr. Lee Li Yuan (Head, Medical department and Clinical Research Centre, Hospital Sri Manjung), Prof. Dr. Rifdy Mohideen (Internist, IMU Batu Pahat), Assoc. Prof. Dr. Velayudhan Menon (Internist, IMU Batu Pahat), Dr. Lee Tong Weng (Family Physician, Ipoh), Prof. Dr. Tong Seng Fah (Family Physician, UKM), Dr. Stanley Chan (Family Physician, IMU Seremban), Assoc. Prof. Dr. Verna Lee Kar Mun (Family Physician, IMU Bukit Jalil), Dr. Jean-Li Lee (Family Medicine Specialist, KK Seremban)

\section{Conflicts of interest}

None.

\section{References}

1. Boriani G, Pettorelli D. Atrial fibrillation burden and atrial fibrillation type: Clinical significance and impact on the risk of stroke and decision making for long-term anticoagulation. Vascul Pharmacol. 2016;83:26-35.

2. Lip GYH, Brechin CM, Lane DA. The global burden of atrial fibrillation and stroke: A systematic review of the epidemiology of atrial fibrillation in regions outside North America and Europe. Chest. 2012;142(6):1489-1498.

3. Kodani E, Atarashi H. Prevalence of atrial fibrillation in Asia and the world. J Arrhythmia. 2012;28(6):330-7.

4. Adderley NJ, Ryan R, Nirantharakumar K, Marshall T. Prevalence and treatment of atrial fibrillation in UK general practice from 2000 to 2016. Heart. 2019;105(1):27-33.

5. Miller PS, Andersson FL, Kalra L. Are cost benefits of anticoagulation for stroke prevention in atrial fibrillation underestimated? Stroke. 2005;36(2):360-366.

6. Freestone B, Rajaratnam R, Hussain N, Lip GY. Admissions with atrial fibrillation in a multiracial population in Kuala Lumpur, Malaysia. Int J Cardiol. 2003;91(2-3):233-238.
7. Guo Y, Lip GYH, Apostolakis S. The unmet need of stroke prevention in atrial fibrillation in the Far East and South East Asia. Malays J Med Sci. 2012;19(3):1-7.

8. Bai Y, Wang YL, Shantsila A, et al. The global burden of atrial fibrillation and stroke: A systematic review of the clinical epidemiology of atrial fibrillation in Asia. Chest. 2017;152(4):810-820.

9. Bungard TJ, Ghali WA, Teo KK, et al. Why do patients with atrial fibrillation not receive warfarin? Arch Intern Med. 2000;160(1):41-6.

10. Buckingham TA, Hatala R. Anticoagulants for atrial fibrillation: Why is the treatment rate so low? Clin Cardiol. 2002;25(10):447-54.

11. Frankel DS, Parker SE, Rosenfeld LE, et al. HRS/NSA 2014 survey of atrial fibrillation and stroke: Gaps in knowledge and perspective, opportunities for improvement. Heart Rhythm. 2015;12(8):e105-e113.

12. Pugh D, Pugh J, Mead GE. Attitudes of physicians regarding anticoagulation for atrial fibrillation: A systematic review. Age Ageing. 2011;40(6):675-83.

13. Peterson G, Boom K, Jackson S, Vial J. Doctors' beliefs on the use of antithrombotic therapy in atrial fibrillation: Identifying barriers to stroke prevention. Intern Med J. 2002;32(1-2):15-23.
14. Murray S, Lazure P, Pullen C, et al. Atrial fibrillation care: challenges in clinical practice and educational needs assessment. Can J Cardiol. 2011;27(1):98-104.

15. Health Technology Assessment Unit. Clinical practice guideline on management of atrial fibrillation. Putrajaya: Ministry of Health Malaysia, 2012.

16. Kirchhof P, Benussi S, Kotecha D, et al. 2016 ESC guidelines for the management of atrial fibrillation developed in collaboration with EACTS. Eur Heart J. 2016;37(38):2893-2962.

17. Robelia P, Kopecky S, Thacher T. Atrial fibrillation care improvement collaborative. BMJ Open Quality. 2015;4:u208947.w3629.

18. Gallagher C, Elliott AD, Wong CX, et al. Integrated care in atrial fibrillation: A systematic review and meta-analysis. Heart. 2017;103(24):1947-1953.

19. van den Dries CJ, van Doorn S, Rutten FH, et al. Integrated management of atrial fibrillation in primary care: Results of the ALL-IN cluster randomized trial [published online ahead of print, 2020 Feb 29]. Eur Heart J. 2020; ehaa055. 


\section{APPENDIX 1}

\section{(A) Questions on knowledge}

Please circle your answers.

1. David Kanang, a 33-year-old Iban male, presents with occasional palpitation. He had rheumatic fever when he was a child. He has an early diastolic murmur with an opening snap that is low pitched at the cardiac apex. There is no late diastolic component to the murmur. He is found to have atrial fibrillation.

Which of the following is the most appropriate long-term drug therapy for him?
A. A novel anticoagulant such as dabigatran
B. Aspirin alone
C. Clopidogrel
D. No need for long-term drug therapy
E. Warfarin

\section{Answer: E}

2. Mr. Tan, a 55-year-old man, comes for a health screening. He is free of chronic disease and is currently asymptomatic. His blood test results are normal, but his ECG shows atrial fibrillation with a heart rate of $88 / \mathrm{min}$. His echocardiogram and thyroid function test done later are all normal.

Which one of the following would be your advice?
A. Aspirin
B. Aspirin plus clopidogrel
C. Clopidogrel
D. No thromboprophylaxis is required
E. Warfarin

\section{Answer: D}

\section{Questions 3 \& 4 are based on the following scenario:}

BK Lim, a 63-year-old man, presents with a history of atrial fibrillation, hypertension, and diabetes. His daughter, who accompanied the patient, states that yesterday, the patient had a period when he could not speak or understand words, and that approximately 4 weeks prior, he staggered against a wall and was unable to stand unaided because of weakness in his legs. She states that both instances lasted approximately half an hour. She was unable to persuade her father to go to the emergency room either time.

Past medical history: Hypertension for 15 years, well-controlled; type 2 diabetes for five years. Medications: Diltiazem CD $300 \mathrm{mg}$ daily; lisinopril $40 \mathrm{mg}$ daily; metformin $500 \mathrm{mg}$ bd; aspirin $75 \mathrm{mg}$ daily.

Tobacco history: Smoked 2 packs a day; quit when he was diagnosed with hypertension. Alcohol history: Drinks 1 beer a day.

Review of systems: Denies dyspnea, dizziness, or syncope; denies focal motor weakness or loss of sensation, except for the reported incident.

Physical exam: Body mass index 26.5kg/m2; BP $134 / 82 \mathrm{~mm} \mathrm{Hg}$; heart rate $88 \mathrm{bpm}$ at rest, irregularly irregular pattern. 
3. What is his CHA2DS2-VASc score?
A. 0
B. 1
C. 2
D. 3
E. 4

\section{Answer: E}

4. Based on his current clinical presentation, what will be your choice of next management strategy?
A. Add a beta-blocker for rate control
B. Add warfarin for thromboprophylaxis
C. Replace aspirin with warfarin for thromboprophylaxis
D. Rhythm control with pharmacological cardioversion
E. Rhythm control with electrical cardioversion

\section{Answer: C}

\section{Questions 5-7 are based on the following scenario:}

Meenachi is a 64-year-old woman with hypertension and a long history of poorly controlled hyperthyroidism who developed atrial fibrillation six months ago. The atrial fibrillation persisted despite treatment with radioactive iodine. There is no history of transient ischemic attack or stroke. She takes 1-2 units of alcohol per day. She is currently on the following medications: amlodipine $5 \mathrm{mg}$ daily, L-thyroxine $0.1 \mathrm{mg}$ daily. Clinically, she is euthyroid, with blood pressure $120 / 70 \mathrm{mmHg}$, heart rate $84 / \mathrm{min}$, BMI $25 \mathrm{~kg} / \mathrm{m} 2$. Her recent full blood count, renal profile, and liver function test are all normal.

5. Which of the following factors is unlikely to increase her bleeding risk if she were to be started on warfarin for thromboprophylaxis?
A. Alcohol intake 1-2 units per day
B. Her body mass index
C. Her systolic blood pressure exceeding $160 \mathrm{mmHg}$
D. Increasing age
E. Occurrence of renal impairment

\section{Answer: B}

6. Meenachi was started on warfarin. What is the target international normalization ratio (INR) that should be aimed for?
A. 0.5 to 1.0
B. 1.0 to 2.0
C. 1.5 to 2.5
D. 2.0 to 3.0
E. 2.5 to 3.5

Answer: D 
7. Which of the following pieces of dietary advice is appropriate for Meenachi?
A. Avoid all green leafy vegetables
B. Avoid all fruits with green skin
C. Can take liberal amounts of cranberry juice
D. Can take liberal amounts of dried prunes
E. Take a consistent amount of green leafy vegetables

\section{Answer: E}

\section{Questions 8-10 are based on the following scenario:}

Mr. JS, a 61-year-old bus driver, presents with a two-week history of mild palpitations associated with mild giddiness without any episodes of fainting. He has had no such episode before. There was no chest pain, orthopnea, or paroxysmal nocturnal dyspnoea.

PMH: No significant medical illness or history of recent bleeding. No past history of asthma or COPD.

Physical examination: Comfortable, not in distress. Pink. No edema is noted.

BP: $130 / 80 \mathrm{mmHg}$; PR 120/min, irregularly irregular, JVP is not raised.

No other abnormalities detected on examination of the peripheral pulses and the precordium. ECG confirms atrial fibrillation with a heart rate of $130 / \mathrm{min}$.

8. Which of the following investigations is least helpful for Mr. JS at this stage?
A. Echocardiogram
B. Exercise stress test
C. Full blood count
D. Serum electrolytes
E. Thyroid function test

\section{Answer: B}

9. Investigations failed to find a specific cause of his atrial fibrillation. What management strategy is appropriate for Mr. JS?
A. Rate control first, then long-term warfarin
B. Rate control plus short-term warfarin, then rhythm control
C. Rhythm control, no need for initial anticoagulant therapy
D. Rate control alone, no need for rhythm control
E. Rate control first, then antiplatelet therapy

\section{Answer: B}

10. What is the first-line drug you would choose to control Mr. JS's heart rate?
A. Amiodarone
B. Amlodipine
C. Digoxin
D. Metoprolol
E. Verapamil

\section{Answer: D}

